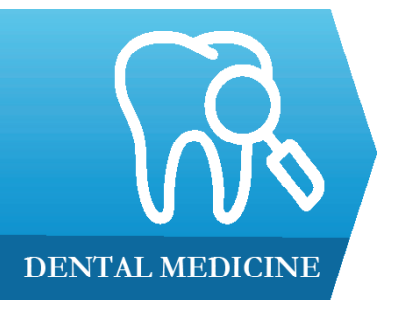

DENTAL MEDICINE

\section{Clinical and radiographic evaluation of demineralized freeze-dried bone allograft with concentrated growth factor versus concentrated growth factor alone in the treatment of intrabony defects}

Tithi Vaid, Santosh Kumar, Rupal Mehta, Sujay Shah, Surabhi Joshi, Susmita Bhakkand, Tanvi Hirani

Department of Periodontology and Implantology, Karnavati School of Dentistry, Gandhinagar, Gujarat, India

\begin{abstract}
Background. Periodontal disease is one of the major causes of alveolar bone loss. There are various ways of regenerating the lost bone, i.e. guided tissue regeneration, bone grafts, and growth factors. In this purview, it becomes immensely important for a clinician to decide the best modality of treatment. In this study, we compared the effect of demineralized freeze-dried bone allograft (DFDBA) in combination with concentrated growth factors (CGF) verses CGF alone.

Methods. This double-blind, split-mouth study was conducted on ten patients with two comparable bilateral intrabony defects. Each pair of defects was randomly treated by DFDBA + CGF or CGF alone. Clinical parameters such as plaque index (PI), modified gingival index (MGI), pocket probing depth (PPD), and relative attachment level (RAL) were recorded at baseline, three months, and six months. In addition, radiograph with grids was also taken at baseline and six months. The paired t-test was used to compare the pre- and post-treatment values and the unpaired t-test was used to compare the test and control group.

Results. The PI score decreased significantly from baseline to six months. Similarly, the mean MGI score decreased significantly from baseline to six months. The intragroup comparison showed that there was a significant reduction in PPD in both the test and control group. However, the intergroup comparison showed that the reduced pocket depth was not significant. The intragroup radiographic comparison showed that there was the significant formation of bone in both the test and control group but inter-group showed that the formation of bone among both the group were non-significant.

Conclusion. Radiographic and clinical outcomes of this study concluded that post six months, both groups demonstrated significant improvement in clinical and radiographic parameters. However, the addition of DFDBA to CGFs did not give any additional benefits.
\end{abstract}

Keywords: allograft, concentrated growth factor, periodontal regeneration

\section{Introduction}

Periodontal diseases encompass

Received in revised form: 15.07.2020

Accepted: 04.08.2020

Address for correspondence:

drsantoshkumar2004@gmail.com

This work is licensed under a Creative Commons Attribution-NonCommercial-

NoDerivatives 4.0 International License a group of inflammatory conditions resulting in pathological alterations of the periodontium, seen as a loss of connective tissue attachment and supporting alveolar bone. Loss of alveolar bone is one of the distinguishing signs of periodontal disease and is thought to represent the anatomical sequel to the apical spread of periodontitis [1]. Various types of bone deformities can result from a periodontal disease such as horizontal and vertical osseous defects $[2,3]$. Vertical or angular defects lead to the formation of osseous defects where the base of the defect is located apical to the surrounding bone. The amount and severity of alveolar 
bone damage in the dentition are usually evaluated by a combination of radiographic and clinical parameters [4]. They are essential adjuncts to the clinician in the diagnosis, treatment planning, and assessment of prognosis of the periodontal patient.

The periodontal disease tends to alter the cementum, resulting in the loss of connective tissue. The relationship between periodontal disease and local microorganisms are very well recognized. It is a universally accepted fact that the removal of pathogenic microorganism present in plaque and calculus is the primary aim of periodontal treatment $[5,6]$. Previously it was thought that bacterial endotoxins could penetrate the cementum of periodontally diseased root surfaces. So the goal of periodontal therapy was to obtain a treated root surface with smooth and hard surface properties [7]. In contrast, several recent studies have suggested that the endotoxins were not located within cementum and removal of necrotic cementum was not necessary for a successful periodontal treatment [7].

Hard tissue destruction caused by periodontitis is often treated by various types of regenerative therapy, which include guided tissue regeneration (GTR), bone grafts, and growth factors. These techniques regenerate the tooth-supporting structures [8]. The GTR therapy has high variability, technique sensitivity, and less predictability of regeneration even as a complete and expectable reconstruction of periodontal tissues is hard to obtain with any therapeutic plan [9]. Typically, the amount of regeneration depends on the regenerative potential of residual periodontal tissues and types of intrabony defects [10-12].

Numerous bone constituents such as autogenous grafts, demineralized freeze-dried bone allografts [13], bovine bone xenografts, or synthetic bone alternatives have established regenerative potential and have been effectively used in the treatment of intrabony defects. The usage of demineralized freeze-dried bone allografts (DFDBA), whether alone or in amalgamation with other treatment modalities for periodontal therapy, has repeatedly demonstrated significant improvements in both soft and hard clinical tissue parameters [14-16].

DFDBA was initially introduced in dentistry in 1965 , for the regeneration of periodontal defects in humans. However, it was clinically utilized in 1975 for the first time [17]. DFDBA offers an osteoconductive surface, and also acts as a significant source of osteoinductive factors. As such, it prompts mesenchymal cell migration, attachment, and osteogenesis when implanted in well-vascularized bone. It also encourages endochondral bone regeneration when surrounded in tissues that would otherwise not form the bone. DFDBA comprises of bone morphogenic proteins (BMPs) such as BMP 2, 4, and 7, which help stimulate osteoinduction. Thus, commercially prepared, allograftretained proteins have the capacity to influence cell behavior in vivo [18].

Polypeptide growth factors, an essential element in tissue engineering, have proven to have an imperative role in the differentiation and growth of cells concerned in the periodontal healing $[19,20]$. Recombinant types of growth factors have shown to have promising effects in various studies but lack practical and clinical implication due to the complex nature of the application and their elevated costs. They are among the first cells to reach the site and initiates the wound healing [21]. The plasma is also the primary source of concentrated growth factors.

Corigliano et al. were the first to develop concentrated growth factors (CGFs) [22]. CGFs are prepared by centrifuging venous blood at alternating and regulated speeds using a medical-grade centrifuge. Altering the centrifugation speeds allow the segregation of a much denser fibrin matrix more abundant in growth factors than typically found in PRP or PRF. It was observed that the fibrin network consisted of thick and thin fibrillar foundations. Numerous platelet cell elements were also seen, creating a cell aggregate stuck among the fibrin network [23]. Rodella et al. proved the occurrence of TGF- $\beta 1$ and VEGF in CGF and red blood cells (RBC) layers, signifying that an enhanced CGF segregation technique could improve the number of growth factors in the CGF layer [23]. Moreover, their results also concluded that an increased number of CD34-positive cells in GCFs - CD34 having been demonstrated to play a vital role in vascular preservation, angiogenesis, and neovascularization [23]. In theory, CGFs seem to show enormous potential for tissue renewal in clinical and biotechnological applications, as evident in a study of sinus and alveolar ridge augmentation [24]. However, there are only rare research works supportive of this theory.

The purpose of this study was to evaluate clinically the reduction in probing depth, gain in clinical attachment level and change in gingival position, and to evaluate radiographically, the osseous defect fill using DFDBA as a bone graft material and CGF.

\section{Methods \\ Study population}

This split-mouth study recruited 10 patients aged from 18 to 60 years suffering from periodontal disease. All the subjects were informed about the nature of the research, and informed consent was obtained prior to their inclusion in the study.

This is a split-mouth study which recruited 10 patients who were diagnosed with generalized chronic periodontitis and met the inclusion and exclusion criteria of the study protocol. The subjects were selected randomly with no discrimination of sex, caste and religion or socioeconomic status. The ethical clearance was obtained by the institutional ethical committee (KSD/2017/34). This study was performed in accordance with the Helsinki Declaration of 1975. Each of the patients was treated in the Department of Periodontology by the same surgeon to reduce the bias.

Patient selection was based on the presence of at least one tooth with a probing depth (PD) of $\geq 6 \mathrm{~mm}$, radiographic evidence of intrabony defect, and also systemically healthy. 
Patients who were unwilling to maintain adequate oral hygiene, pregnant and lactating females, patients with a habit of smoking or consuming tobacco products and the patients who have undergone periodontal surgery within a year were excluded from the study.

\section{Study design}

All the patients underwent full mouth scaling and root planing, and oral hygiene instructions were given. After periodontal revaluation, the patients were inducted in this study. At the time of surgery, the patient's mouth was disinfected using $0.2 \%$ chlorhexidine mouthwash for 60 seconds. The area to undergo surgery was anaesthetized with $2 \%$ lignocaine hydrochloride $(1: 80,000)$ solution. Intra crevicular incision was placed using a BP handle No. 3 And blade No. 15. Full-thickness mucoperiosteal periodontal flaps were reflected on buccal and lingual site. The granulation tissue was removed using Gracey curettes (Hu-Friedy, Chicago, IL, USA), and the roots were thoroughly scaled and planed using hand and ultrasonic instruments. Once the debridement of the defect was done, direct measurement of the osseous defect was done with the help of UNC-15 probe. The vertical bone depth, bone defect width and the number of bony walls present were noted.

On the test site (Group A), pre-suturing was done using 3.0 silk suture followed by bony defect filling with DFDBA (Tata Memorial Hospital, Mumbai, Maharashtra, India) in combination with CGF. The pre-sutured mucoperiosteal flaps were repositioned and secured with a figure of eight technique. The surgical area was isolated with a non-eugenol (Coe-pack, USA) periodontal pack.

At the control site (Group B), pre-suturing was done using 3.0 silk suture followed by CGF placement in the bony defect. As previously mentioned, the surgical procedure was completed, and the patient was recalled for follow up after 10 days. All the postoperative instructions were given. All the patients were prescribed Amoxicillin $500 \mathrm{mg}$ TDS for 3 days and Diclofenac sodium $400 \mathrm{mg}$ TDS for 3 days.

\section{Clinical measurements}

All baseline parameters were recorded on the day of surgery by the same periodontist who was blinded to the type of treatment received, in all the patients. Patients recall visits were scheduled at 3 months and 6 months' time and the measurements were recorded by the same examiner. The parameters recorded were Plaque Index (PI, Turesky-Gilmore-Glickman Modification of QuigleyHein Plaque Index-1970), Modified gingival Index (MGI, Lobene-1986), Pocket probing depth (PPD), and relative attachment level (RAL).

\section{CGF Preparation}

Nine $\mathrm{ml}$ of venous blood was withdrawn in sterile Vacuette tube ${ }^{\circledR}$ (Greiner Bio-One, GmbH, Kremsmunster, Austria) without any anticoagulant solutions. The venous blood was immediately centrifuged for $2 \mathrm{~min}$ at 2700 rpm, 4 min at $2400 \mathrm{rpm}, 4 \mathrm{~min}$ at $2700 \mathrm{rpm}$, followed by $3 \mathrm{~min}$ at $3000 \mathrm{rpm}$. At the end of the centrifugation, three blood layers were created in the test tube. The middle layer consisting of huge and dense polymerized fibrin block containing the CGFs were used in surgery.

\section{Stent fabrication}

Acrylic stents were prepared and were confirmed for their stability. It was used to standardize the measurement of periodontal pocket depth. The calibrated clinician who was not involved in the treatment procedures performed all the measurements at baseline, 3 months and 6 months' time after surgery.

\section{Radiographic measurements}

Radiovisiographs (RVG) were taken with the help of Rinn XCP (Dentsply Sirona, Charlotte, North Carolina, USA) system by the standardized paralleling technique using a standard intraoral grid. The area of the defect was calculated manually. The radiographic parameters were recorded as baseline and 6 months postoperatively.

Anatomic parameters, as identified by Eickholz et al. [25], were considered for radiographic analysis (Figure 1):

1. Cemento-enamel Junction (CEJ)

2. Alveolar crest (AC)

3. The base of the defect (BD)

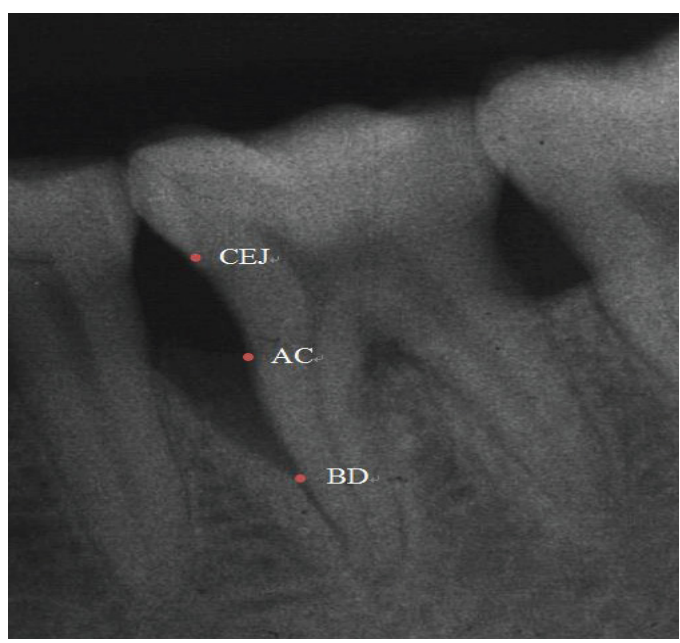

Figure 1. Radiographic landmarks.

The base of the defect (BD) - the distances from the cement-enamel junction (CEJ) to the most in-depth extension of the bony defect.

Alveolar crest (AC) - the distance from the cementenamel junction $(\mathrm{CEJ})$ to the alveolar crest.

AUX I - An auxiliary line is drawn in the direction of tooth axis.

AUX II - $2^{\text {nd }}$ auxiliary line perpendicular to the tooth axis was drawn through the most coronal extension of the lateral wall of the intrabony defect.

INFRA 1- Distance from CEJ to BD - Distance 
from CEJ to AC (Difference of distances from CEJ to BD and $\mathrm{CEJ}$ to $\mathrm{AC}$ )

INFRA 2 - distance from the point where AUX II crossed the contour of the root to BD.

$\mathrm{BDW}=$ distance measured from lateral margin of intrabony defect to the point where AUX II cross the root surface.

Linear Bone Growth $=\mathrm{CEJ}$ to $\mathrm{BD}$ at baseline $-\mathrm{CEJ}$ to $\mathrm{BD}$ after 6 months

Area of defect $=1 / 2($ INFRA $1 * \mathrm{BDW})$

Bone fill $\%=($ Linear bone growth $/$ Defect depth $)$ *100 (Figure 2)

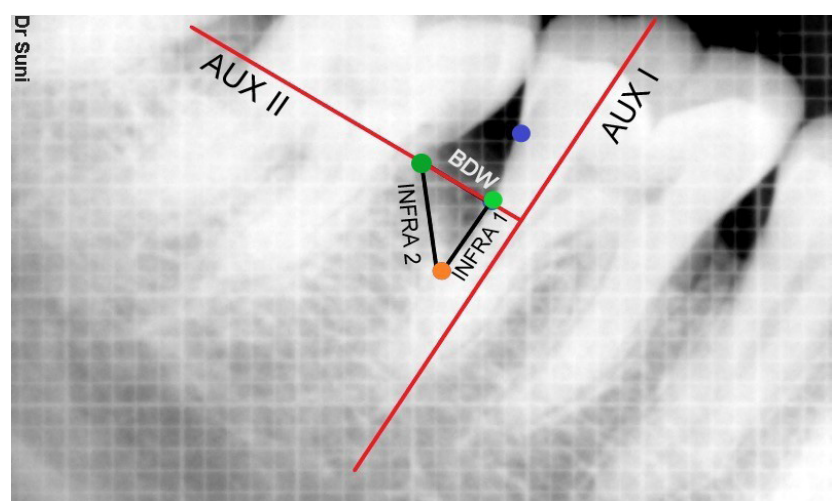

Figure 2. Radiographic landmarks for calculation.

\section{Results}

This split-mouth study was done to evaluate clinical and radiographic parameters between the test group in which DFDBA and CGF as compared to the control group in which CGF alone used in the treatment of intrabony defect.

The mean Plaque index scores recorded at baseline and 6 months were $0.70 \pm 0.18$ and $0.44 \pm 0.16$ respectively. The PI score decreased significantly from baseline to 6-month time. Similarly, the mean MGI score at baseline and 6 months were $1.48 \pm 0.213$ and $0.51 \pm 0.123$, respectively. The MGI score decreased significantly from baseline to 6-month time (Table I).

Table I. Mean plaque index and modified gingival index at baseline and 6 month post surgery.

$\begin{array}{lccc} & \text { Mean Value } & \text { P-value } \\ \text { Paseline } & \text { Plaque Index } \\ \text { 6-month post-surgery } & 0.70 & 0.44 \\ \text { Modified gingival index } & 0.02 * \\ \text { Baseline } & 1.48 & 0.00 * \\ \text { 6-month post-surgery } & 0.51 & \\ \text { * Significant } & & \end{array}$

The pocket probing depth (in $\mathrm{mm}$ ) for the test group was $7.00 \pm 1.054$ at baseline, which was decreased to 4.50 \pm 1.08 at 3 months and $3.20 \pm 0.63$ at 6 months. Hence the intragroup comparison showed a significant reduction in probing depth from baseline to 3 months, baseline to 6 months. In the control group, the pocket probing depth (in $\mathrm{mm}$ ) recorded at baseline, 3 months and 6 months were $6.40 \pm 0.966,4.50 \pm 0.972$ and $3.40 \pm 0.699$ respectively, showing a mean difference of $1.900,3.000$ and 1.100 from baseline to 3 months, baseline to 6 months, 3 months to 6 months with $P$ values of $0.002,0.000$ and 0.225 respectively (Table II).

Table II. Intragroup comparison of pocket probing depth values (in $\mathrm{mm}$ ) of test and control sites at baseline, 3 months and 6 months.

\begin{tabular}{l|l|c|c|c|c|c} 
Group & $\begin{array}{l}\text { Intra } \\
\text { group }\end{array}$ & $\mathbf{N}$ & Mean & SD & MD & p \\
\hline \multirow{5}{*}{ Test } & Baseline & 10 & 7.00 & 1.054 & 2.50 & $0.000^{*}$ \\
& 3 months & 10 & 4.50 & 1.080 & & \\
& Baseline & 10 & 7.00 & 1.054 & 3.80 & $0.000^{*}$ \\
& 6 months & 10 & 3.20 & 0.632 & & \\
& 3 months & 10 & 4.50 & 1.080 & 1.30 & 0.092 \\
& 6 months & 10 & 3.20 & 0.632 & & \\
Con- & Baseline & 10 & 6.40 & 0.966 & 1.90 & $0.002 *$ \\
trol & 3 months & 10 & 4.50 & 0.972 & & \\
& Baseline & 10 & 6.40 & 0.966 & 3.00 & $0.000 *$ \\
& 6 months & 10 & 3.40 & 0.699 & & \\
& 3 months & 10 & 4.50 & 0.972 & 1.10 & 0.225 \\
& 6 months & 10 & 3.40 & 0.699 & &
\end{tabular}

*Significant; SD - Std. Deviation; MD - Mean Difference

On the intergroup comparison between the test and control group after t-test, the probing depth was statically insignificant at baseline as the P-value was 0.827 . However, at 3 months and 6 months, the probing depth reduction was statically more significant in the test group as compared to control groups with $\mathrm{P}$ values of 1.000 and 0.999 , respectively (Table III).

Table III. Intergroup comparison of pocket probing depth values (in $\mathrm{mm}$ ) of test and control sites, baseline, 3 months, 6 months.

\begin{tabular}{l|l|c|c|c|c|c}
$\begin{array}{l}\text { Time } \\
\text { period }\end{array}$ & Group & $\mathbf{N}$ & Mean & SD & MD & $\mathbf{p}$ \\
\hline Base line & Test & 10 & 7.00 & 1.054 & 0.600 & 0.827 \\
& Control & 10 & 6.40 & 0.966 & & \\
\multirow{2}{*}{3 months } & Test & 10 & 4.50 & 1.080 & 0.000 & 1.000 \\
& Control & 10 & 4.50 & 0.972 & -0.200 & 0.999
\end{tabular}

SD - Std. Deviation; MD - Mean Difference

The relative attachment level (in $\mathrm{mm}$ ) for the test group was $8.10 \pm 1.197$ at baseline, which was decreased to $4.70 \pm 0.483$ at 3 months and $3.60 \pm 0.699$ at 6 months. Hence the intragroup comparison showed significantly reduced in probing depth from baseline to 3 months, baseline to 6 months. In the control group, the relative attachment level (in $\mathrm{mm}$ ) recorded at baseline, 3 months and 6 months were $7.70 \pm 0.949,5.10 \pm 1.370$ and 4.30 
\pm 1.059 respectively, showing a mean difference of 2.600 , 3.400 and 0.800 from baseline to 3 months, baseline to 6 months, 3 months to 6 months with P values of $0.000,0.000$ and 0.675 respectively (Table IV).

Table IV. Intragroup comparison of relative attachment level (in $\mathrm{mm}$ ) of test and control sites at baseline, 3 months and 6 months.

\begin{tabular}{|l|l|c|c|c|c|c}
\hline Group & $\begin{array}{l}\text { Intra } \\
\text { group }\end{array}$ & $\mathbf{N}$ & Mean & SD & MD & p \\
\hline \multirow{5}{*}{ Test } & Baseline & 10 & 8.10 & 1.197 & 3.400 & $0.000^{*}$ \\
& 3 months & 10 & 4.70 & 0.483 & & \\
& Baseline & 10 & 8.10 & 1.197 & 4.500 & $0.000^{*}$ \\
& 6 months & 10 & 3.60 & 0.699 & & \\
& 3 months & 10 & 4.70 & 0.483 & 1.100 & 0.322 \\
& 6 months & 10 & 3.60 & 0.699 & & \\
Con- & Baseline & 10 & 7.70 & 0.949 & 2.600 & $0.000^{*}$ \\
trol & 3 months & 10 & 5.10 & 1.370 & & \\
& Baseline & 10 & 7.70 & 0.949 & 3.400 & $0.000^{*}$ \\
& 6 months & 10 & 4.30 & 1.059 & & \\
& 3 months & 10 & 5.10 & 1.370 & 0.800 & 0.675 \\
& 6 months & 10 & 4.30 & 1.059 & &
\end{tabular}

*Significant; SD - Std. Deviation; MD - Mean Difference

On the intergroup comparison between the test and control group after t-test, the probing depth was statically insignificant at baseline as the P-value was 0.827 . However, at 3 months and 6 months, the relative attachment level reduction was statically more significant in the test group as compared to control groups with $\mathrm{P}$ values of 0.976 and 0.786 respectively (Table $\mathrm{V}$ ).

Table V. Intergroup comparison relative attachment level values (in $\mathrm{mm}$ ) of test and control sites at baseline, 3 months and 6 months.

\begin{tabular}{ll|c|c|c|c|c}
$\begin{array}{l}\text { Time } \\
\text { period }\end{array}$ & Group & N & Mean & SD & MD & p \\
\hline Base line & Test & 10 & 8.10 & 1.197 & 0.400 & 0.976 \\
& Control & 10 & 7.70 & 0.949 & & \\
3 months & Test & 10 & 4.70 & 0.483 & -0.400 & 0.976 \\
& Control & 10 & 5.10 & 1.370 & & \\
\multirow{2}{*}{6 months } & Test & 10 & 3.60 & 0.699 & -0.700 & 0.786 \\
& Control & 10 & 4.30 & 1.059 & &
\end{tabular}

SD - Std. Deviation; MD - Mean Difference

On intragroup comparison of the area of defect at baseline (Figure 3a) and 6 months (Figure 3b) mean value was $9.50 \pm 1.76$ and $5.67 \pm 1.490 \mathrm{~mm}^{2}$ respectively, which was found to statically significant in the test group. Whereas in the control group the mean value at baseline (Figure 4a) and 6 months (Figure 4b) was $10.55 \pm 3.022$ and $6.35 \pm 2.517 \mathrm{~mm}^{2}$ respectively which was found to statically significant (Table VI).
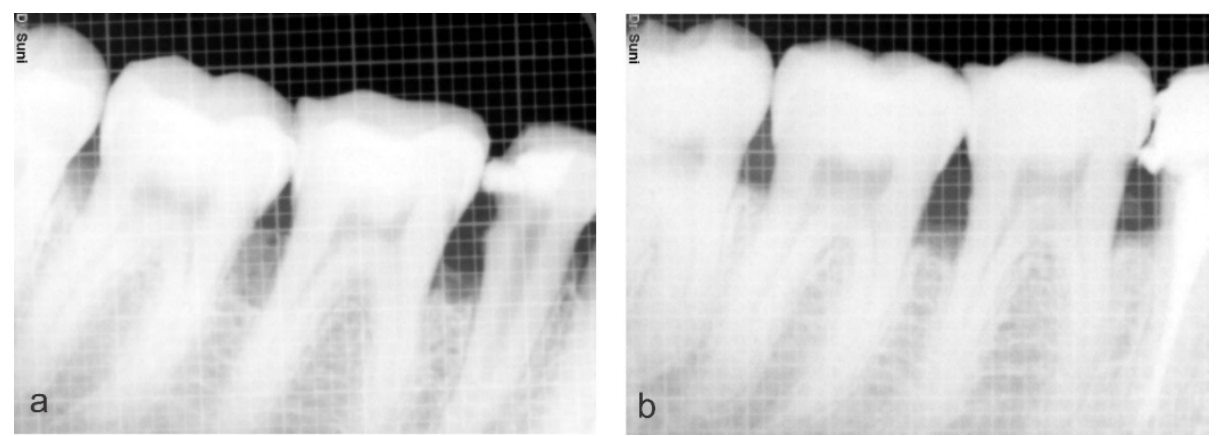

Figure 3. a) Preoperative radiograph showing the defect; b) Postoperative radiograph showing the bone formation on the test site (Group A).
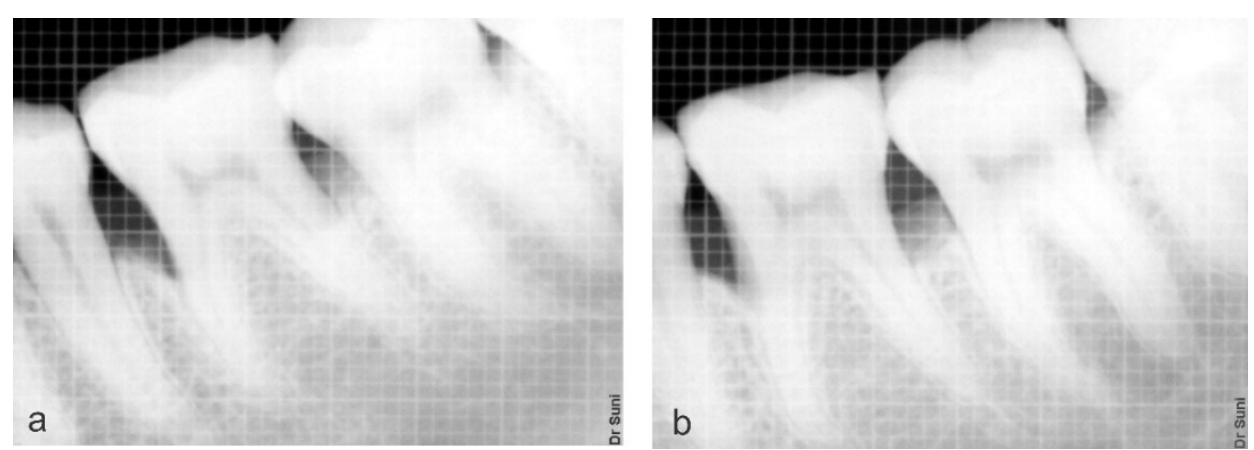

Figure 4. a) Preoperative radiograph showing the defect; b) Postoperative radiograph showing the bone formation on Control site (Group B). 
Table VI. Intragroup comparison of radiographic area of defect values (in $\mathrm{mm}^{2}$ ) of test and control sites at baseline and 6 months.

\begin{tabular}{l|l|c|c|c|c|c} 
Group & $\begin{array}{l}\text { Intra } \\
\text { group }\end{array}$ & $\mathbf{N}$ & Mean & SD & MD & p \\
Test & Baseline & 10 & 9.50 & 1.763 & 3.825 & $0.007^{*}$ \\
Con- & 6 months & 10 & 5.67 & 1.490 & & \\
trol & 6 moseline & 10 & 10.55 & 3.022 & 4.200 & $0.003^{*}$
\end{tabular}

*Significant; SD - Std. Deviation; MD - Mean Difference

On intergroup comparison, after t-test, the area of defect at baseline between the test and control groups was statically insignificant as the P-value was 0.787 . However, the decrease in the area of defect was more significant as compared to the control group at 6 months but statically insignificant reduction between test and control group at 6 months (Table VII).

Table VII. Intergroup comparison of area of defect (in $\mathrm{mm}^{2}$ ) at baseline and 6 months.

\begin{tabular}{ll|c|c|c|c|c}
$\begin{array}{l}\text { Time } \\
\text { period }\end{array}$ & Group & $\mathbf{N}$ & Mean & SD & MD & p \\
Baseline & Test & 10 & 9.500 & 1.763 & -1.050 & 0.787 \\
& Control & 10 & 10.550 & 3.022 & & \\
6 months & Test & 10 & 5.675 & 1.490 & -0.675 & 0.932 \\
& Control & 10 & 6.350 & 2.517 & & \\
D - Std. Deviation; MD - Mean Difference
\end{tabular}

\section{Discussion}

This study compared the combination of CGFs + DFDBA with CGFs alone in the treatment of periodontal intrabony defects. The results indicated that both the group gave similar results in improving clinical and radiographic parameters. The aim of periodontal therapy is to restore the periodontal tissue lost as a result of periodontal diseases [26]. Periodontal regeneration is the phenomenon which response to different stimuli. It is a natural process requiring coordinated responses, involving the formation of new periodontal ligament fibers, bone and the cementum. Periodontitis degrades different parts of the periodontium which ultimately leads to tooth loss. Reconstruction of the periodontal structures lost as a consequence of periodontal diseases has been an evasive goal for the more than a century [27].

In the current study, although there was no significant difference between the two treatment modalities, but a significant pocket-depth reduction, intrabony defect fill, and clinical attachment gain were achieved. Many results have been obtained from various studies using a different commercial brand of DFDBA. This can be due to the activity and concentration of BMP in the different allograft, that makes the result unpredictable. The apparent source for the allografts is the cadavers, and the younger cadavers have the highest levels of BMP [28]. Moreover, membranous and cortical bones have higher concentrations of BMP than trabecular and endochondral bone, respectively [29]. Particle size also plays a significant role in bone formation. The particle size of more than $250 \mu \mathrm{m}$ enhances bone formation [30]. In addition, the methods of sterilization by ethylene oxide or irradiation affects the amount of BMP and DFDBA properties. There is also histological evidence that DFDBA supports the formation of a new attachment apparatus in intrabony defects, whereas OFD results in periodontal repair characterized primarily by the establishment of a long junctional epithelial attachment [31]. To summarize, the fabrication and different processing steps have a vital role in the osteoconductive properties of DFDBA $[32,33]$.

Hormonal fluctuations in female patients may alter the status of periodontal health and affect the treatment outcome. The most pronounced periodontal changes occur during pregnancy and lactation. Treatment consideration for pregnant patients with periodontal disease may include deferral of periodontal surgery until after parturition [34]. Hence, pregnant and lactating women were not part of the study.

Concentrated growth factors (CGF) is a modified form of PRF prepared by repeatedly switching the centrifugation speed and are characterized as a relatively rigid fibrin clot [35]. The fibrin buffy coat is a significant component in CGFs. Use of different centrifugation speeds permits the collection of abundant growth factors located just below the buffy coat and above the dense clot portion [36]. CGF releases numerous growth factors such as Platelet-derived growth factor (PDGF), Transforming growth factor- $\beta 1$ (TGF- $\beta 1$ ) and $\beta 2$ (TGF- $\beta 2$ ), Fibroblast growth factor (FGF), Vascular endothelial growth factor (VEGF), Brain-derived growth factor (BDGF) and Insulinlike growth factor (IGF) which stimulate cell proliferation, matrix remodelling and angiogenesis [37,38].

In the present study, the plaque index scores were significantly decreased from baseline to 6 months. The minimum amount of plaque did not interfere with the regenerative process and all the patients maintained good oral hygiene throughout the study period. The decline in plaque index scores over the duration of the study was observed due to the repeated reinforcement of oral hygiene habits in recall visits and overall general improvement in periodontal parameters. A similar trend was found in the reduction of plaque index scores in a study done by Gothi et al. [39], who compared FDBA and DFDBA graft material to determine their efficacy in intrabony defects.

The pocket probing depth in the test group was 7.00 \pm 1.054 at baseline, which decreased to $4.50 \pm 1.08$ at 3 months and $3.20 \pm 0.63$ at 6 months. In the control group, the PPD (in $\mathrm{mm}$ ) recorded at baseline, 3 months and 6 months were $6.40 \pm 0.966,4.50 \pm 0.972$ and $3.40 \pm 0.699$ respectively. Hence the intragroup comparison showed a significant decrease in probing depth from baseline to 3 months, baseline to 6 months and from 3 months to 6 
months. This finding correlates to a study by Pradeep et al. which showed statistically as well as clinically significant similar results in terms of PD reduction [40]. A different study with similar results was conducted by Gothi et al. [39] who compared DFDBA and FDBA in intrabody defects. The results showed a decrease in probing depth from baseline to six months.

Reduction of pocket depth and gain of clinical attachment is the major clinical outcome measurements used to determine the success of treatment. In the past, analyses of pocket depth were the only technique to measure periodontitis. However, in merely probing the pocket and recording the pocket depth is inadequate as a surge in periodontal pocket depth could be due to a gingival enlargement without obliteration of underlying periodontal tissue. In a few cases, the recession of marginal gingival may accompany attachment loss, concealing ongoing disease progression if the pocket depth measurement is taken unaided.

The relative attachment level in both groups showed improvement over the duration of the study. The intragroup comparison showed significantly decreased probing depth from baseline to 6 months. Similar results were observed in a comparative study of open flap debridement (OFD) and PRF and the OFD + DFDBA by Shah et al [41]. There were significant improvements in RAL at the end of 6 months for both groups. Statistically, a significant difference was noted in RAL at 6 months for both groups.

Radiography is the only non-invasive method for evaluating hard tissue changes and are permanent records. Following successful therapy of periodontal diseases, both increase in bony density and gain of bony support may be observed on the radiograph. Radiovisiographs were taken for all the defect sites using the Intraoral millimeter grid by long cone paralleling technique to standardize the projection geometry in order to measure the radiographic area of bone fill. Radiographs were taken at baseline and at 6 months of each defect site.

Parameters showing bone formation such as the area of defect, linear bone growth (LBG), percentage of bone fill and area of defect were measured at 6 months and calculated manually, as complete bone maturation and mineralization do not occur by the third month. The area of defect at baseline was $9.50 \pm 1.763$ for the test group, which significantly reduced to $5.67 \pm 1.490$ at 6 months. For the control group area of defect at baseline was $10.55 \pm 3.022$ which significantly reduced to $6.35 \pm 2.517$ at 6 months. On intergroup comparison after t-test, the area of defect at baseline between the test and control groups was statically insignificant. However, the decreased in area of defect was greater as compared to the control group at 6 months but statically insignificant reduction between test and control group at 6 months. A similar result was obtained by the study done by Gupta et al. [42] and Gothi et al [39]. The above results were also similar to a comparative study with enamel matrix derivative (EMD) and demineralised freezedried bone allografts (DFDBA) with DFDBA alone for the treatment of human periodontal intrabony defects study done by Aspriello et al. [43] and Jaykumar et al [44].

To summarize, in the present study, an overall favorable response was observed as no patient-reported of any post-operative complication other than those considered as normal following the periodontal surgery. Also, no antigenic reactions were observed in any of the patients, indicating the safety of the material used. All the clinical and radiographic parameters recorded in both the study groups showed improvement; however, better periodontal regeneration and wound healing was observed with the use of DFDBA and CGF.

\section{Conclusion}

By comparing the radiographic and clinical outcome of regenerative therapy using DFDBA + CGF and CGF alone, this study showed that post six months, both groups demonstrated significant improvement in clinical and radiographic parameters. However, the addition of DFDBA to CGFs did not give any added advantage.

\section{References}

1. Könönen E, Gursoy M, Gursoy UK. Periodontitis: A Multifaceted Disease of Tooth-Supporting Tissues. J Clin Med. 2019;8:1135.

2. Salaria SK, Ghuman SK, Kumar S, Sharma G. Management of localized advance loss of periodontal support associated Grade II furcation and intrabony defect in chronic periodontitis patient through amalgamation of platelet-rich fibrin and hydroxyapatite bioactive glass composite granules. Contemp Clin Dent. 2016;7:405-408.

3. Josephi J, Reddy KK, Seshan H, Reddy V, Reddy J, Nera M, et al. Clinical and Radiographic Evaluation of Platelet Rich Plasma in Combination with Demineralised Freeze-Dried Bone Allograft in the Treatment of Periodontal Intrabony Defects: A Comparative Study. Adv Hum Biol. 2015;5:5665.

4. Preshaw PM. Detection and diagnosis of periodontal conditions amenable to prevention. BMC Oral Health. 2015;15 Suppl 1:S5.

5. Gupta S, Jain P, Kumra M, Rehani S, Mathias Y, Gupta R, et al. Bacterial Viability within Dental Calculus: An Untrodden, Inquisitive Clinico-Patho- Microbiological Research. J Clin Diagn Res. 2016;10:ZC71-ZC75. doi:10.7860/ JCDR/2016/18783.8192

6. Mombelli A. Microbial colonization of the periodontal pocket and its significance for periodontal therapy. Periodontol 2000. 2018;76:85-96.

7. Bozbay E, Dominici F, Gokbuget A, Cintan S, Guida L, Aydin MS, et al. Preservation of root cementum: a comparative evaluation of power-driven versus hand instruments. Int J Dent Hyg. 2018;16:202-209.

8. Artzi Z, Tal H, Platner O, Wasersprung N, Weinberg E, 
Slutzkey S, et al. Deproteinized bovine bone in association with guided tissue regeneration or enamel matrix derivatives procedures in aggressive periodontitis patients: a 1-year retrospective study. J Clin Periodontol. 2015;42:547-556.

9. Ausenda F, Rasperini G, Acunzo R, Gorbunkova A, Pagni G. New Perspectives in the Use of Biomaterials for Periodontal Regeneration. Materials (Basel). 2019;12:2197.

10. Susin C, Fiorini T, Lee J, De Stefano JA, Dickinson DP, Wikesjö UM. Wound healing following surgical and regenerative periodontal therapy. Periodontol 2000. 2015;68:83-98.

11. Zheng C, Chen J, Liu S, Jin Y. Stem cell-based bone and dental regeneration: a view of microenvironmental modulation. Int J Oral Sci. 2019;11(3):23.

12. Rojas MA, Marini L, Pilloni A, Sahrmann P. Early wound healing outcomes after regenerative periodontal surgery with enamel matrix derivatives or guided tissue regeneration: a systematic review. BMC Oral Health. 2019;19:76.

13. Lopes D, Martins-Cruz C, Oliveira MB, Mano JF. Bone physiology as inspiration for tissue regenerative therapies. Biomaterials. 2018;185:240-275.

14. Jain D, Deepa D. A comparative evaluation of freeze-dried bone allograft with and without bioabsorbable guided tissue regeneration membrane Healiguide ${ }^{\circledR}$ in the treatment of Grade II furcation defects: A clinical study. J Indian Soc Periodontol. 2015;19:645-650.

15. Agarwal A, Gupta ND. Platelet-rich plasma combined with decalcified freeze-dried bone allograft for the treatment of noncontained human intrabony periodontal defects: a randomized controlled split-mouth study. Int J Periodontics Restorative Dent. 2014;34:705-711.

16. Pierannunzii L, Zagra L. Bone grafts, bone graft extenders, substitutes and enhancers for acetabular reconstruction in revision total hip arthroplasty. EFORT Open Rev. 2016;1:431-439.

17. Grassi FR, Grassi R, Vivarelli L, Dallari D, Govoni M, Nardi GM, et al. Design Techniques to Optimize the Scaffold Performance: Freeze-dried Bone Custom-made Allografts for Maxillary Alveolar Horizontal Ridge Augmentation. Materials (Basel). 2020;13:1393.

18. Shigeyama Y, D'Errico JA, Stone R, Somerman MJ. Commercially-prepared allograft material has biological activity in vitro. J Periodontol. 1995;66:478-487.

19. Lynch SE, Williams RC, Polson AM, Howell TH, Reddy MS, Zappa UE, et al. A combination of platelet-derived and insulin-like growth factors enhances periodontal regeneration. J Clin Periodontol. 1989;16:545-548.

20. Giannobile WV, Finkelman RD, Lynch SE. Comparison of canine and non-human primate animal models for periodontal regenerative therapy: results following a single administration of PDGF/IGF-I. J Periodontol. 1994;65:11581168.

21. Eisinger F, Patzelt J, Langer HF. The Platelet Response to Tissue Injury. Front Med (Lausanne). 2018;5:317. doi:10.3389/fmed.2018.00317

22. Choukroun J, Diss A, Simonpieri A, Girard MO, Schoeffler C, Dohan SL, et al. Platelet-rich fibrin (PRF): a second- generation platelet concentrate. Part V: histologic evaluations of PRF effects on bone allograft maturation in sinus lift. Oral Surg Oral Med Oral Pathol Oral Radiol Endod. 2006;101:299-303.

23. Rodella LF, Favero G, Boninsegna R, Buffoli B, Labanca M, Scarì G, et al. Growth factors, CD34 positive cells, and fibrin network analysis in concentrated growth factors fraction. Microsc Res Tech. 2011;74:772-777.

24. Sohn D-S, Heo J-U, Kwak D-H, et al. Bone regeneration in the maxillary sinus using an autologous fibrin-rich block with concentrated growth factors alone. Implant Dent. 2011;20(5):389-395. doi:10.1097/ID.0b013e31822f7a70

25. Eickholz P, Hörr T, Klein F, Hassfeld S, Kim TS. Radiographic parameters for prognosis of periodontal healing of infrabony defects: two different definitions of defect depth. J Periodontol. 2004;75:399-407.

26. Hägi TT, Laugisch O, Ivanovic A, Sculean A. Regenerative periodontal therapy. Quintessence Int. 2014;45:185-192.

27. Caton JG, Greenstein G. Factors related to periodontal regeneration. Periodontol 2000. 1993;1:9-15.

28. Li H, Pujic Z, Xiao Y, Bartold PM. Identification of bone morphogenetic proteins 2 and 4 in commercial demineralized freeze-dried bone allograft preparations: pilot study. Clin Implant Dent Relat Res. 2000;2:110-117.

29. Moreira CA, Dempster DW, Baron R. Anatomy and Ultrastructure of Bone - Histogenesis, Growth and Remodeling. In: Feingold KR, Anawalt B, Boyce A, et al., eds. Endotext. MDText.com, Inc.; 2000. Available from: http:/www.ncbi.nlm.nih.gov/books/NBK279149/

30. Schwartz Z, Mellonig JT, Carnes DL Jr, de la Fontaine J, Cochran DL, Dean DD, et al. Ability of commercial demineralized freeze-dried bone allograft to induce new bone formation. J Periodontol. 1996;67:918-926.

31. Bowers GM, Chadroff B, Carnevale R, Mellonig J, Corio R, Emerson J, et al. Histologic evaluation of new attachment apparatus formation in humans. Part III. J Periodontol. 1989;60:683-693.

32. Lynch SE, ed. Tissue Engineering: Applications in Oral and Maxillofacial Surgery and Periodontics. 2. ed. Quintessence Publ; 2008: p. 1-312.

33. Resnik RR, ed. Misch's Contemporary Implant Dentistry. Fourth edition. Elsevier; 2020.

34. Parameter on Periodontitis Associated With Systemic Conditions. J Periodontol. 2000;71 Suppl 5S:876-879.

35. Masuki H, Okudera T, Watanebe T, Suzuki M, Nishiyama $\mathrm{K}$, Okudera $\mathrm{H}$, et al. Growth factor and pro-inflammatory cytokine contents in platelet-rich plasma (PRP), plasma rich in growth factors (PRGF), advanced platelet-rich fibrin (A-PRF), and concentrated growth factors (CGF). Int $\mathrm{J}$ Implant Dent. 2016;2:19.

36. Qiao J, Duan J, Zhang Y, Chu Y, Sun C. The effect of concentrated growth factors in the treatment of periodontal intrabony defects. Future Sci OA. 2016;2:FS136.

37. Yu B, Wang Z. Effect of concentrated growth factors on beagle periodontal ligament stem cells in vitro. Mol Med Rep. 2014;9:235-242.

38. Schär MO, Diaz-Romero J, Kohl S, Zumstein MA, Nesic 
D. Platelet-rich concentrates differentially release growth factors and induce cell migration in vitro. Clin Orthop Relat Res. 2015;473:1635-1643. doi:10.1007/s11999-015-4192-2

39. Gothi R, Bansal M, Kaushik M, Khattak B, Sood N, Taneja $\mathrm{V}$. A comparative evaluation of freeze dried bone allograft and decalcified freeze dried bone allograft in the treatment of intrabony defects: A clinical and radiographic study. J Indian Soc Periodontol. 2015;19:411-415.

40. Pradeep AR, Bajaj P, Rao NS, Agarwal E, Naik SB. PlateletRich Fibrin Combined With a Porous Hydroxyapatite Graft for the Treatment of 3-Wall Intrabony Defects in Chronic Periodontitis: A Randomized Controlled Clinical Trial. J Periodontol. 2017;88:1288-1296.

41. Shah M, Patel J, Dave D, Shah S. Comparative evaluation of platelet-rich fibrin with demineralized freeze-dried bone allograft in periodontal infrabony defects: A randomized controlled clinical study. J Indian Soc Periodontol.
2015;19:56-60. doi:10.4103/0972-124X.145803

42. Gupta G. Clinical and radiographic evaluation of intra-bony defects in localized aggressive periodontitis patients with platelet rich plasma/hydroxyapatite graft: A comparative controlled clinical trial. Contemp Clin Dent. 2014;5:445451.

43. Aspriello SD, Ferrante L, Rubini C, Piemontese M. Comparative study of DFDBA in combination with enamel matrix derivative versus DFDBA alone for treatment of periodontal intrabony defects at 12 months post-surgery. Clin Oral Investig. 2011;15:225-232.

44. Jayakumar A, Rajababu P, Rohini S, Butchibabu K, Naveen A, Reddy PK, et al. Multi-centre, randomized clinical trial on the efficacy and safety of recombinant human plateletderived growth factor with $\beta$-tricalcium phosphate in human intra-osseous periodontal defects. J Clin Periodontol. 2011;38:163-172. 